\title{
The existence of best proximity points with the weak $P$-property
}

\section{Tomonari Suzuki*}

\section{${ }^{*}$ Correspondence:}

suzuki-t@mns.kyutech.ac.jp

Department of Basic Sciences,

Kyushu Institute of Technology,

Tobata, Kitakyushu 804-8550, Japan

\begin{abstract}
We improve some existence theorem of best proximity points with the weak $P$-property, which has been recently proved by Zhang et al.

MSC: Primary 54H25; secondary 54E50

Keywords: best proximity point; the weak P-property; the Banach contraction principle; Kannan's fixed point theorem; completion
\end{abstract}

\section{Introduction}

Let $(A, B)$ be a pair of nonempty subsets of a metric space $(X, d)$, and let $T$ be a mapping from $A$ into $B$. Then $x \in A$ is called a best proximity point if $d(x, T x)=d(A, B)$, where $d(A, B)=\inf \{d(x, y): x \in A, y \in B\}$. We have proved many existence theorems of best proximity points. See, for example, [1-6]. Very recently, Caballero et al. [7] proved a new type of existence theorem, and Zhang et al. [8] generalized the theorem. The theorem proved in [8] is Theorem 8 with an additional assumption of the completeness of $B$. The essence of the result in [7] becomes very clear in [8], however, we have not learned the essence completely.

Motivated by the fact above, in this paper, we improve the result in [8]. Also, in order to consider the discontinuous case, we give a Kannan version.

\section{Preliminaries}

In this section, we give some preliminaries.

Definition 1 Let $(A, B)$ be a pair of nonempty subsets of a metric space $(X, d)$, and define $A_{0}$ and $B_{0}$ by

$$
A_{0}=\{x \in A: \text { there exists } u \in B \text { such that } d(x, u)=d(A, B)\}
$$

and

$$
B_{0}=\{u \in B: \text { there exists } x \in A \text { such that } d(x, u)=d(A, B)\} .
$$

Then

- (Sankar Raj [9]) $(A, B)$ is said to have the P-property if $A_{0} \neq \varnothing$ and the following holds:

$$
x, y \in A_{0}, u, v \in B_{0}, \quad d(x, u)=d(y, v)=d(A, B) \quad \Longrightarrow \quad d(x, y)=d(u, v) .
$$

o2013 Suzuki; licensee Springer. This is an Open Access article distributed under the terms of the Creative Commons Attribution License (http://creativecommons.org/licenses/by/2.0), which permits unrestricted use, distribution, and reproduction in any medium, provided the original work is properly cited. 
- (Zhang et al. [8]) $(A, B)$ is said to have the weak P-property if $A_{0} \neq \varnothing$ and the following holds:

$$
x, y \in A_{0}, u, v \in B_{0}, \quad d(x, u)=d(y, v)=d(A, B) \quad \Longrightarrow \quad d(x, y) \leq d(u, v) .
$$

Proposition 2 Let $(A, B)$ be a pair of nonempty subsets of a metric space $(X, d)$, and define $A_{0}$ and $B_{0}$ by (1) and (2). Assume that $A_{0} \neq \varnothing$. Then the following are equivalent:

(i) $(A, B)$ has the weak P-property.

(ii) The conjunction of the following holds:

(ii-1) For every $u \in B_{0}$, there exists a unique $x \in A_{0}$ with $d(x, u)=d(A, B)$.

(ii-2) There exists a nonexpansive mapping $Q$ from $B_{0}$ into $A_{0}$ such that $d(Q u, u)=d(A, B)$ for every $u \in B_{0}$.

Proof We note that $B_{0} \neq \varnothing$ because $A_{0} \neq \varnothing$. First, we assume (i). Let $x, y \in A_{0}$ and $u \in B_{0}$ satisfy $d(x, u)=d(y, u)=d(A, B)$. Then from (i), we have

$$
d(x, y) \leq d(u, u)=0
$$

thus, $x=y$. So (ii-1) holds. We put $Q u=x$. Then from the definition of the weak $P$-property, we have $d(Q u, Q v) \leq d(u, v)$ for $u, v \in B_{0}$, that is, $Q$ is nonexpansive. Conversely, we assume (ii). Let $x, y \in A_{0}$ and $u, v \in B_{0}$ satisfy $d(x, u)=d(y, v)=d(A, B)$. Then from (ii-1), we have $Q u=x$ and $Q v=y$. Therefore,

$$
d(x, y)=d(Q u, Q v) \leq d(u, v)
$$

holds.

Lemma 3 Let $(A, B)$ be a pair of subsets of a metric space $(X, d)$, and define $A_{0}$ and $B_{0}$ by (1) and (2). Assume that $A_{0} \neq \varnothing$. Let $T$ be a mapping from $A$ into $B$, and let $Q$ be a mapping from $B_{0}$ into $A_{0}$ such that $d(Q u, u)=d(A, B)$ for every $u \in B_{0}$. Then the following holds:

$$
\left\{u_{n}\right\} \subset B_{0}, \quad \lim _{n \rightarrow \infty} u_{n}=w, \quad T\left(\lim _{n \rightarrow \infty} Q u_{n}\right)=w \quad \Longrightarrow \quad w \in B_{0} .
$$

Proof Let $\left\{u_{n}\right\}$ be a sequence in $B_{0}$ such that $\left\{u_{n}\right\}$ converges to $w \in X$, and $T\left(\lim _{n} Q u_{n}\right)=w$. We put $y=\lim _{n} Q u_{n}$. Since $T y=w$, we have $y \in A$ and $w \in B$. Since

$$
d(y, w)=\lim _{n \rightarrow \infty} d\left(Q u_{n}, u_{n}\right)=d(A, B)
$$

we have $y \in A_{0}$ and $w \in B_{0}$.

Lemma 4 Let $(X, d)$ be a metric space, let $A, A_{0}, B_{0}$ be nonempty subsets such that $A$ is complete and $A_{0} \subset A$. Let $T$ be a mapping from $A$ into $X$ such that $T\left(A_{0}\right) \subset B_{0}$, and let $Q$ be a nonexpansive mapping from $B_{0}$ into $A_{0}$. Let $\bar{Q}$ be the mapping whose graph $\operatorname{Gr}(\bar{Q})$ is the completion of $\operatorname{Gr}(Q)$. Assume (3). Then the following hold:

(i) $\bar{Q}$ is well-defined and nonexpansive.

(ii) $\bar{Q} w=z$ is equivalent to that there exists a sequence $\left\{u_{n}\right\}$ in $B_{0}$ such that $\lim _{n} u_{n}=w$ and $\lim _{n} Q u_{n}=z$. 
(iii) The domain of $\bar{Q}$ is $\overline{B_{0}}$, where $\overline{B_{0}}$ is the completion of $B_{0}$.

(iv) The range of $\bar{Q}$ is a subset of $\overline{A_{0}}$, where $\overline{A_{0}}$ is the completion of $A_{0}$.

(v) $T \circ \bar{Q} w=w$ implies $T \circ Q w=w$.

(vi) $\bar{Q} \circ T z=z$ implies $Q \circ T z=z$.

(vii) The range of $\bar{Q}$ is a subset of $A$.

Proof We consider that the whole space is the completion of $X$. Since $Q$ is Lipschitz continuous, $\bar{Q}$ is well-defined. The rest of (i) and (ii)-(iv) are obvious. By using (3), we can easily prove (v) and (vi). From the completeness of $A$, we obtain (vii).

\section{Fixed point theorems}

In this section, we give fixed point theorems, which are used in the proofs of the main results.

Theorem 5 Let $(X, d)$ be a metric space, let $A, A_{0}, B_{0}$ be nonempty subsets such that $A$ is complete and $A_{0} \subset A$. Let $T$ be a contraction from $A$ into $X$ such that $T\left(A_{0}\right) \subset B_{0}$, and let $Q$ be a nonexpansive mapping from $B_{0}$ into $A_{0}$. Assume (3). Then $Q \circ T$ has a unique fixed point in $A_{0}$.

Proof We consider that the whole space is the completion of $X$. Define a nonexpansive mapping $\bar{Q}$ as in Lemma 4 . Since $T$ is continuous, $T\left(\overline{A_{0}}\right)$ is a subset of $\overline{B_{0}}$. Let $S$ be the restriction of $T$ to $\overline{A_{0}}$. Then $\bar{Q} \circ S$ is a contraction on $\overline{A_{0}}$. So the Banach contraction principle yields that there exists a unique fixed point $z$ of $\bar{Q} \circ S$ in $\overline{A_{0}}$. Since $\bar{Q} \circ T z=z$, by Lemma 4(vi), $z$ is a fixed point of $Q \circ T$.

\section{Remark}

- If $X=A=A_{0}=B_{0}$ and $Q$ is the identity mapping on $B_{0}$, then Theorem 5 becomes the Banach contraction principle [10].

- We can prove Theorem 5 with the mapping $T \circ \bar{Q}$ as in the proof of Theorem 7 .

We prove generalizations of Kannan's fixed point theorem [11].

Theorem 6 Let $(X, d)$ be a metric space, let $Y$ be a complete subset of $X$, and let $T$ be a mapping from $Y$ into $X$. Assume that the following hold:

(i) There exists $\alpha \in[0,1 / 2)$ such that $d(T x, T y) \leq \alpha d(x, T x)+\alpha d(y, T y)$ for all $x, y \in Y$.

(ii) There exists a nonempty subset $Z$ of $Y$ such that $T(Z) \subset Z$.

Then there exists a unique fixed point $z$, and for every $x \in Z,\left\{T^{n} x\right\}$ converges to $z$.

Proof Fix $x \in Z$. Then from the proof in Kannan [11], we obtain that $\left\{T^{n} x\right\}$ converges to a fixed point, and the fixed point is unique.

Remark If $X=Y=Z$, then Theorem 6 becomes Kannan's fixed point theorem [11].

Using Theorem 6, we obtain the following.

Theorem 7 Let $(X, d)$ be a metric space, let $A, A_{0}, B_{0}$ be nonempty subsets such that $A$ is complete and $A_{0} \subset A$. Let $T$ be a mapping from $A$ into $X$ such that $T\left(A_{0}\right) \subset B_{0}$, and let $Q$ be a nonexpansive mapping from $B_{0}$ into $A_{0}$. Assume that (3) and the following hold: 
- There exist $\alpha \in[0,1 / 2)$ and $\mu \in[0, \infty)$ such that

$$
d(T x, T y) \leq \alpha(d(x, T x)-\mu)+\alpha(d(y, T y)-\mu)
$$

for $x, y \in A$ and $d(Q u, u) \leq \mu$ for all $u \in B_{0}$.

Then $T \circ Q$ has a unique fixed point in $B_{0}$.

Proof We consider that the whole space is the completion of $X$. Define a nonexpansive mapping $\bar{Q}$ as in Lemma 4. From the continuity of $d, d(\bar{Q} u, u) \leq \mu$ for $u \in \overline{B_{0}}$. For $u, v \in \overline{B_{0}}$, we have

$$
\begin{aligned}
& d(T \circ \bar{Q} u, T \circ \bar{Q} v) \\
& \quad \leq \alpha(d(\bar{Q} u, T \circ \bar{Q} u)-\mu)+\alpha(d(\bar{Q} v, T \circ \bar{Q} v)-\mu) \\
& \quad \leq \alpha(d(\bar{Q} u, u)+d(u, T \circ \bar{Q} u)-\mu)+\alpha(d(\bar{Q} v, v)+d(v, T \circ \bar{Q} v)-\mu) \\
& \quad \leq \alpha d(u, T \circ \bar{Q} u)+\alpha d(v, T \circ \bar{Q} v) .
\end{aligned}
$$

Hence $T \circ \bar{Q}$ is a Kannan mapping from $\bar{B}_{0}$ into $X . T \circ \bar{Q}\left(B_{0}\right)=T \circ Q\left(B_{0}\right) \subset B_{0}$ is obvious. So by Theorem 6, there exists a unique fixed point $w$ of $T \circ \bar{Q}$ in $\overline{B_{0}}$. By Lemma $4(\mathrm{v}), w \in B_{0}$ and $w$ is a fixed point of $T \circ Q$.

\section{Remark}

- Since $T$ is not necessarily continuous, the range of $T \circ \bar{Q}$ is not necessarily included by $\overline{B_{0}}$. Because of the same reason, we cannot prove Theorem 7 with the mapping $\bar{Q} \circ T$.

- It is interesting that we do not need the completeness of any set related to $B_{0}$ directly.

Of course, we need the completeness of $A$.

\section{Main results}

In this section, we give the main results.

Theorem 8 (Zhang et al. [8]) Let $(A, B)$ be a pair of subsets of a metric space $(X, d)$, and define $A_{0}$ and $B_{0}$ by (1) and (2). Let $T$ be a contraction from $A$ into $B$. Assume that the following hold:

(i) $(A, B)$ has the weak P-property.

(ii) $A$ is complete.

(iii) $T\left(A_{0}\right) \subset B_{0}$.

Then there exists a unique $z \in A$ such that $d(z, T z)=d(A, B)$.

Proof By Proposition 2(ii-2), there exists a nonexpansive mapping $Q$ from $B_{0}$ into $A_{0}$ such that $d(Q u, u)=d(A, B)$ for every $u \in B_{0}$. Then by Lemma 3 , all the assumptions in Theorem 5 hold. So there exists a unique fixed point $z$ of $Q \circ T$ in $A_{0}$. This implies that $d(z, T z)=d(A, B)$. Let $x \in A$ satisfy $d(x, T x)=d(A, B)$. Then from Proposition 2(ii-1), $x \in A_{0}$, $T x \in B_{0}$ and $Q \circ T x=x$ hold. Since $Q \circ T$ has a unique fixed point, we obtain $x=z$. Hence $z$ is unique. 


\section{Remark}

- If we weaken (i) to the conjunction of $A_{0} \neq \varnothing$ and (ii-2) in Proposition 2, we obtain only the existence of best proximity points.

- In [8], we assume the completeness of $B$.

- Exactly speaking, in [8], we obtained a theorem connected with Geraghty's fixed point theorem [12]. However, in this paper, the difference between the two fixed point theorems is not essential. This means that we can easily modify Theorem 8 to be connected with Geraghty's theorem.

Theorem 9 Let $(A, B)$ be a pair of subsets of a metric space $(X, d)$, and define $A_{0}$ and $B_{0}$ by (1) and (2). Let $T$ be a mapping from $A$ into B. Assume that (i)-(iii) in Theorem 8 and the following hold:

(iv) There exists $\alpha \in[0,1 / 2)$ such that

$$
d(T x, T y) \leq \alpha(d(x, T x)-d(A, B))+\alpha(d(y, T y)-d(A, B))
$$

$$
\text { for } x, y \in A \text {. }
$$

Then there exists a unique $z \in A$ such that $d(z, T z)=d(A, B)$.

Proof By Proposition 2(ii-2), there exists a nonexpansive mapping $Q$ from $B_{0}$ into $A_{0}$ such that $d(Q u, u)=d(A, B)$ for every $u \in B_{0}$. Then by Theorem 7, there exists a unique fixed point $w$ of $T \circ Q$ in $B_{0}$. This implies that $d(z, T z)=d(A, B)$, where $z=Q w$. Let $x \in A$ satisfy $d(x, T x)=d(A, B)$. Then from Proposition 2(ii-1), $x \in A_{0}, T x \in B_{0}$ and $Q \circ T x=x$ hold. Since $T \circ Q \circ T x=T x$, we have $T x=w$, and hence $x=Q \circ T x=Q w=z$. Therefore, $z$ is unique.

Remark If we weaken (i) to the conjunction of $A_{0} \neq \varnothing$ and (ii-2) in Proposition 2, we obtain only the existence of best proximity points.

\section{Additional result}

In this section, we give a proposition similar to Proposition 2.

Proposition 10 Let $(A, B)$ be a pair of nonempty subsets of a metric space $(X, d)$, and define $A_{0}$ and $B_{0}$ by (1) and (2). Assume that $A_{0} \neq \varnothing$. Then the following are equivalent:

(i) $(A, B)$ has the P-property.

(ii) The conjunction of the following holds:

(ii-1) For every $u \in B_{0}$, there exists a unique $x \in A_{0}$ with $d(x, u)=d(A, B)$.

(ii-2) There exists an isometry $Q$ from $B_{0}$ onto $A_{0}$ such that $d(Q u, u)=d(A, B)$ for every $u \in B_{0}$.

Proof We note $B_{0} \neq \varnothing$. First, we assume (i). Let $x, y \in A_{0}$ and $u \in B_{0}$ satisfy $d(x, u)=$ $d(y, u)=d(A, B)$. Then from (i), we have $d(x, y)=d(u, u)=0$, thus, $x=y$. So (ii-1) holds. We put $Q u=x$. Then it is obvious that $Q$ is isometric. For every $x \in A_{0}$, there exists $u \in B_{0}$ with $d(x, u)=d(A, B)$. From (ii-1), $Q u=x$ obviously holds, and hence $Q$ is surjective. Conversely, we assume (ii). Let $x, y \in A_{0}$ and $u, v \in B_{0}$ satisfy $d(x, u)=d(y, v)=d(A, B)$. Then we have $Q u=x$ and $Q v=y$. Therefore, $d(x, y)=d(Q u, Q v)=d(u, v)$ holds. 


\section{Competing interests}

The author declares that he has no competing interests.

\section{Acknowledgements}

The author is supported in part by the Grant-in-Aid for Scientific Research from the Japan Society for the Promotion of Science.

Received: 24 June 2013 Accepted: 18 September 2013 Published: 07 Nov 2013

References

1. Alghamdi, MA, Shahzad, N, Vetro, F: Best proximity points for some classes of proximal contractions Abstr. Appl. Anal. 2013, Article ID 713252 (2013)

2. Di Bari, C, Suzuki, T, Vetro, C: Best proximity points for cyclic Meir-Keeler contractions. Nonlinear Anal. 69, 3790-3794 (2008)

3. Eldred, AA, Kirk, WA, Veeramani, P: Proximal normal structure and relatively nonexpansive mappings. Stud. Math. 171, 283-293 (2005)

4. Eldred, AA, Veeramani, P: Existence and convergence of best proximity points. J. Math. Anal. Appl. 323, 1001-1006 (2006)

5. Suzuki, T, Kikkawa, M, Vetro, C: The existence of best proximity points in metric spaces with the property UC. Nonlinear Anal. 71, 2918-2926 (2009)

6. Vetro, C: Best proximity points: convergence and existence theorems for $p$-cyclic mappings. Nonlinear Anal. 73 2283-2291 (2010)

7. Caballero, J, Harjani, J, Sadarangani, K: A best proximity point theorem for Geraghty-contractions. Fixed Point Theory Appl. 2012, Article ID 231 (2012)

8. Zhang, J, Su, Y, Cheng, Q: A note on 'A best proximity point theorem for Geraghty-contractions'. Fixed Point Theory Appl. 2013, Article ID 99 (2013)

9. Sankar Raj, V: Banach's contraction principle for non-self mappings. Preprint

10. Banach, S: Sur les opérations dans les ensembles abstraits et leur application aux équations intégrales. Fundam. Math. 3, 133-181 (1922)

11. Kannan, R: Some results on fixed points. II. Am. Math. Mon. 76, 405-408 (1969)

12. Geraghty, MA: On contractive mappings. Proc. Am. Math. Soc. 40, 604-608 (1973)

10.1186/1687-1812-2013-259

Cite this article as: Suzuki: The existence of best proximity points with the weak $P$-property. Fixed Point Theory and

Applications 2013, 2013:259

\section{Submit your manuscript to a SpringerOpen ${ }^{\ominus}$ journal and benefit from:}

- Convenient online submission

- Rigorous peer review

Immediate publication on acceptance

- Open access: articles freely available online

- High visibility within the field

- Retaining the copyright to your article 